\title{
Señalización celular en el hipocampo epiléptico
}

\author{
I. Ferrer
}

\section{CELL SIGNALING IN THE EPILEPTIC HIPPOCAMPUS}

\begin{abstract}
Summary. Cell signaling commanding death or survival in human epileptic hippocampus is difficult to trace because of the long interval between the beginning of symptoms and the sampling of damaged cerebral tissue for neuropathological examination. Intraperitoneal injection of the glutamate analogue kainic acid (KA) is a useful tool to analyze the effects of seizures and the excitotoxic damage in the rodent hippocampus. KA acts on NMDA and KA receptors, whereas it has little impact on AMPA receptors. Neurons of the hilus and CA3 neurons are primary targets of $K A$, although parvalbumin-containing GABAergic neurons are less vulnerable than glutamatergic neurons. Immediate responses to KA are hsp-70 mRNA induction and HSP-70/72 protein expression, as well as c-fos and c-jun mRNA, and c-Fos and c-Jun protein expression in the hippocampus. Yet increased c-Fos and c-Jun expression is not a predictor of cell death or cell survival. In contrast, the tissular plasminogen activator (tPA) and the membrane Fas/Fas-L signaling pathway probably have a role in facilitating cell death following KA injection. The involvement of other pathways remains controversial. Increased expression of the pro-apoptotic Bax together with decreased Bcl-2 suggests Baxmediated apoptosis. Activation of the mitochondrial pathway includes leakage of citochrome $c$ to the cytosol and activation of the caspase cascade leading to apoptosis. However, other studies have emphasized the limited expression of caspase-3, the main executioner of apoptosis, and the relevance of necrosis as the main form of cell death following KA excitotoxicity. Phosphorylationdependent activation of several kinases, including MAPK, $p$-38 and JNK/SAPK, and their substrates has been found in KA-treated animals. Decreased CREBp expression is associated with cell death whereas increased ATF-2P and Elk-1P are associated with cell survival. Trophic factors probably do not play a significant role during the early stages of hippocanmpal damage but they are important in the remodeling of the granukle cells and the sprouting of mossy fibers to the molecular layer of the dentate gyrus. This abnormal regeneration, in turn, facilitates seizure recruitment and the chronic maintenance of convulsions. [REV NEUROL 2002; 34: 544-50] Key words. Apoptosis. Bcl-2. Caspases. Fas. HSP-72. Kainic acid. Kinases. Necrosis. Seizures.
\end{abstract}

Clásicamente se conoce la esclerosis temporal mesial como una causa frecuente de epilepsia tonicoclónica generalizada. Las lesiones asociadas a ésta consisten en una pérdida neuronal y astrocitosis en la capa piramidal de las regiones CA1, CA2 y CA3 del hipocampo y de subpoblaciones neuronales del hilio de la circunvolución dentada. Las neuronas granulares de la circunvolución dentada se conservan mejor. Las lesiones no se limitan al hipocampo propio, ya que existe pérdida neuronal en la corteza parahipocámpica y fenómenos de rebrote axonal de las fibras musgosas a la zonainternadelacapamoleculardelacircunvolucióndentada[1].

No se conoce bien el fenómeno de inicio lesional en la esclerosis mesial porque el examen neuropatológico tiene lugar en fases frecuentemente muy avanzadas de la historia clínica, en la que se han sucedido múltiples episodios con daño neuronal acumulativo. Sin embargo, el estudio cuidadoso de cerebros epilépticos obtenidos postmortem, asícomo de las muestras de resección quirúrgica realizadas en personas con epilepsia resistente a tratamiento farmacológico, ha permitido una aproximación a los fenómenos residuales del hipocampo epiléptico. Ello incluye el examen de subpoblaciones neuronales susceptibles y resistentes, el estudio de expresión de receptores de neurotransmisores y el desarrollo de fenómenos plásticos, particularmente en la circun-

Recibido: 27.09.01. Acepta dotrasrevisiónexternasinmodificaciones:05.11.01. Unidadde Neuropatología. Serviciode AnatomíaPatológica.HospitalPríncipes de España. Universidad de Barcelona. Hospitalet de Ll., Barcelona, España.

Correspondencia: Dr. Isidro Ferrer. Unidad de Neuropatología. Servicio de Anatomía Patológica. Hospital Príncipes de España. Feixa Llarga, $s / n$. E-08907L'HospitaletdeLl., Barcelona.E-mail:iferrer@sakma.es

Presentado en el I Congreso de la Liga Española contra la Epilepsia, celebrado en Bilbao del 14 al 17 de noviembre de 2001.

(C) 2001, REVISTADENEUROLOGÍA volución dentada, los cuales provocan circuitos excitadores que favorecen la progresión de la epilepsia.

El estudio de hipocampos epilépticos humanos no permite, sin embargo, conocer la secuencia de acontecimientos quellevan ala pérdida neuronal y a la regulación de los fenómenos plásticos. Por ese motivo se han diseñado diferentes modelos animales utilizando estimulaciones eléctricas ofarmacológicas, entre las que seencuentralaadministración sistémica del análogo del glutamatoácidokaínico.

\section{HIPOCAMPOEPILÉPTICOHUMANO}

Un aspecto importante en el daño epiléptico es la afectación selectiva de subpoblaciones neuronales. En el hilio de la circunvolución dentada, las neuronas inmunorreactivas para somatostatina, neuropéptido Y y sustanciaP son particularmente vulnerables $[2,3]$. Por el contrario, las neuronas que contienen las proteínas ligantes de calcio parvoalbúmina o calbindina D28k, resultan ser resistentes. Las neuronas granulares de la circunvolución dentada y las interneuronas de CA 1 y CA3 son inmunorreactivas para calbindina D28k. Además, las neuronas no piramidales del hipocampoinmunorreactivas para parvoalbúmina aparecen conservadas. Estos datos sugieren que la expresión de proteínas ligantes de calcio protege las células frente a la entrada masiva de calcio [4]. Estos datos concuerdan con la conservación de neuronas de hipocampoinmunorreactivas para decarboxilasa delácido glutámicoenelhipocampoepiléptico humano [5].Porotra parte, se ha descrito una conservación de las neuronas hilares que contienen acetilcolinesterasa y conservación de esta actividad enzimática en las fibras de la zona interna de la capa molecular de la circunvolución dentada. Sin embargo, existe una pérdida de acetilcolinesterasa en elhipocampo propioy en la zona externa de lacapa molecular de la circunvolución dentada [6]. 
El estudio de receptores para neurotransmisores ha mostrado unareducción de receptores $\mathrm{GABA}_{\mathrm{A}}(\mathrm{GABA}$-adrenérgicos)mediante autorradiografía e inmunohistoquímica. También se ha observado una reducción de receptores N-metil-D-aspartato (NMDA) y de receptores kaínico en CA3, CA1 e hilio, pero aumento de la expresión en la circunvolución parahipocampal, lo que sugiere circuitos aberrantes excitadores en el hipocampo epiléptico humano. La reducción de receptores y modificaciones en el ligamiento de los receptores NMDA en el hipocampo propio se ha comprobado en otros estudios, en los que, paralelamente, se ha encontrado un aumento de ligamiento a receptores del ácido amino-metil-propiónico(AMPA) en la capa molecular de la circunvolución dentada [7-15].

La pérdida de neuronas hilares lleva consigo una pérdida de dianas para las fibras granulares que parece activar un rebrote de fibras musgosas a la zona interna de la capa molecular de la circunvolución dentada [16-19]. Esto se acompaña de un aumento de expresión de la forma activa de la molécula de adhesión celular neural (PSA-NCAM), que interviene en procesos de migración neural,crecimiento dendríticoy ramificación axonal.Laaparición defenómenos plásticos depende, en parte, de señalizaciones con factores tróficos y sus receptores específicos. Este aspecto tiene repercusiones terapéuticas en el caso de tratamiento con factores tróficos. La expresión del factor neurotrófico derivado del cerebro(BDNF) se modifica en la esclerosis mesial y elBDNF aumenta la actividad de sinapsis excitadoras en el hipocampo epiléptico. El posible efecto beneficioso de BDNFresulta dudoso [21-27].

\section{EXCITOTOXICIDAD CON ÁCIDO KAÍNICO. VÍAS DE SEÑALIZACIÓN CELULAR}

La administración sistémica (por ejemplo, inyección intraperitoneal) de ácido kaínico, un análogo del glutamato, en dosis convulsionantes, produce daño excitotóxico en las neuronas piramidales del hipocampo propio y en el hilio de la circunvolución dentada, pero no en las neuronas granulares de ésta. El daño depende de la dosis, de la especie y de la cepa de los animales, pero el resultado es la muerte de las neuronas en las regiones vulnerables, la proliferación de astrocitos y el aumento de fibras gliales.Porese motivo, el paradigmade administración sistémica deácido kaínico se ha considerado un buen modelo para elestudio de las convulsiones tonicoclónicas generalizadas, cuyo sustrato neuroanatómico es la esclerosis temporal mesial [28-34].

El tratamiento previo con MK-801, un antagonista no competitivo de receptores NMDA, reduce las convulsiones y el daño neuronal, lo que sugiere la implicación de receptores NMDA en el daño inducido por ácido kaínico [35-37]. Por el contrario, la administración de NBQX, un antagonista de receptores AMPA, parece tener poco o nulo efecto sobre el daño excitotóxico.Puesto que NBQX tiene alta afinidad por AMPA pero muy poca por receptores cainato, puede inferirse que la lesión por ácido kaínico se produce por actuación del ácido kaínico sobre receptores específicos con implicación extendida a receptores NMDA [38-40].

La mayor concentración de receptores cainato se encuentra en las neuronas de la región CA3 del hipocampo. También se ha descrito la activación sináptica de receptores cainato en interneuronas (neuronas inhibidoras) del hipocampo [41-44]. Lapresencia de receptores de glutamato GluR6 en las sinapsis de las fibras musgosas parece mediar la actuación de ácido kaínico, ya que los ratones nulos para GluR6 son más resistentes al daño excitotóxico [45]. Asimismo se ha señalado la activación de interneuronasen distintas regiones del hipocampo, que pareceestar mediada, en parte, por receptores GluR5 [46]. La generación de convulsiones tonicoclónicas generalizadas a consecuencia de la estimulación por ácido kaínico depende de receptores cainato, pero en particular de la activación repetitiva de las fibras aferentes y de la inhibición tónica de las interneuronas. El destino final del sistema sometido a una activación doble-de las neuronas excitadoras glutamatérgicas (neuronas piramidales o de proyección) y de neuronas inhibidoras gabérgicas (neuronas no piramidales o de circuito-local)- puede dar lugar a la entrada masiva de calcio en las células diana y desencadenar la muerte celular por necrosis [47-51].

Tanto las neuronas excitadoras como las inhibidoras son susceptibles al ataque excitotóxico, si bien la presencia de parvoalbúmina, una proteína con capacidad de actuar como tampón de las entradas masivas de calcio al interior de las neuronas, confiere un mayor grado de resistencia a las interneuronas frente al ataque excitotóxico $[52,53]$.

Uno de los primeros cambios después de la administración de ácido kaínico es la inducción de ARN mensajero (ARNm) para hsp-70 y la expresión de la proteína de estrés de calor de 70 kDa(HSP-70/72) en las poblaciones neuronales sensibles del hipocampo propio, pero no de la circunvolución dentada. La expresión de esta chaperonina parece tener la función de prevenir el plegamiento anormal de proteínas nacientes en las poblaciones vulnerables al ácido kaínico. En los días siguientes, y durante dos semanas, HSP-70/72 se transporta por el árbol dendrítico y a lo largo de los axones hacia las zonas más distales. HSP 70/72 tiene un papel protector, aunque no consigue rescatar por sí misma a las células de la muerte excitotóxica. La sobreexpresión de HSP-70in vivo protege del daño excitotóxico. Niveles excesivamente altos de HSP-72 pueden ser nocivos para las células [52-58].

También, de modo temprano, existe inducción de ARNm de c-Fos y c-Jun en las regiones vulnerables del hipocampo propio y en la circunvolución dentada después de tres a cinco horas de la inyección de ácido kaínico [59,60]. Por lo que se refiere a la transcripción a proteína, se encuentra un aumento de expresión del factor de transcripción c-Fos a tres horas en el hipocampo propio y en la circunvolución dentada. La inmunorreactividad parac-Fos decrece a las seis horas en la circunvolución dentada, pero permanece alta en el hipocampo propio. Ello ha sugerido que la muerte celular puede asociarse a niveles altos y mantenidos de expresión de c-Fos, pero también se ha demostrado que el incremento prolongado de c-Fos no tiene un carácter predictivo y no se requiere para que se produzca daño neuronal excitotóxico [36,61-64]. También se encuentra un aumento de la expresión de c-Jun en el hipocampo propio y en la circunvolución dentada durante 24 horas después del ataque excitotóxico. El significado delaumentodec-Jun resultacontradictorio, yaquesehaseñalado como marcador de muerte celular retardada secundaria a convulsiones epilépticas, o como un posible marcador de supervivencia neuronal frente al ataque excitotóxico $[60,63,65,66]$.

Respecto a la señalización a través de la membrana celular, es interesante conocer que la serina proteasa extracelular, activador tisulardelplasminógeno(tPA), parecesernecesaria para producir muerte celular, ya que ratones nulos para tPA o para plasminógeno son relativamente resistentes al daño excitotóxico. Este efecto parece mediado por la interacción de tPA con laminina, una proteína de la matriz extracelular [67-70]. 
También relacionado con la señalización a través de la membrana celular se encuentra un aumento de la expresión de Fas-L, ligando específico del receptor Fas, en el hipocampo y en las células granulares de la circunvolución dentada tres horas después de la inyección de ácido kaínico [71]. Mientras que en ésta última región la expresión de Fas-L decrece seis horas después de lainyección de ácido kaínico, lainmunorreactividad paraFas-L se mantiene en el hipocampo propio. Este aspecto resulta relevante, ya que la unión de Fas-L y Fas activa el dominio de muerte de Fas, al que se une FADD, activando a su vez a caspasa-8. La activación de caspasa-8 actúa sobre las caspasas efectoras, quienes ejecutan la muerte por apoptosis. La utilización de ratones nulos para Fas aporta datos sustanciales para conocer el papel del sistema Fas/Fas-L en la señalización de muerte celular excitotóxica. Estudios preliminares con ratones $\mathrm{Fas}^{-/}$sometidos a inyección intraperitoneal de ácido kaínico han mostrado convulsiones, pero menor daño excitotóxico en las regiones diana, lo que indica un papel mediador de la vía de señalización Fas/Fas-Len este modelo de daño excitotóxico. Sin embargo, la presencia de Fas y de Fas-L no es suficiente para desencadenar la cascada de muerte celular por apoptosis porque las neuronas del hipocampo propio mueren, mientras que las neuronas de la circunvolución dentada sobreviven [Ferrer I, Domínguez I, Puig B, datos no publicados].

El papel que desempeñan los miembros de la familia Bcl-2 resulta todavía poco claro. Un estudio preliminar mostró reducción de proteína Bcl-2 y aumento de ARNm para Bax en el hipocampo del ratón después de la inyección sistémica de ácido kaínico [72]. Estudios más precisos han encontrado, mediante Northern blot, una inducción del ARNm de Bax - pero no de Bcl-2 y Bcl-x-desde seis a 24 horas en el hipocampo de ratas inyectadas con ácido kaínico. La expresión de las proteínas Bcl-2, Bcl-x y Bax en el hipocampo propio, analizada mediante Western blot e inmunohistoquímica, es parecida en las células destinadas a morir y en las células que sobreviven [73]. Algunos estudios han mostrado aumento de la expresión de Bax en el hipocampo después del estímulo excitotóxico, y han especulado que Bax, agente proapoptótico, podría desempeñar un papel en la muerte excitotóxica. Sin embargo, estudios de hibridación in situ y de inmunohistoquímica han mostrado inducción de ARNm de Bax y de proteína Bax en el hipocampo propio, pero también en la circunvolución dentada, que es resistente al daño excitotóxico [73].

Es posible quelos efectos de los miembros delafamilia Bcl-2 no dependan de cambios globales de las proteínas, perosí su localización subcelular.Laseñalización demuerteapoptóticaporla vía mitocondrial se desencadena porunaunión de Bax a la membrana mitocondrial y por una liberación de citocromo c al citosol. Esta liberación comporta unión a Apaf-1 en presencia de ATP y activacióndelacaspasa-9, quien, a su vez, activa distintas caspasasefectoras oejecutoras. Elmodoenque se produce la salidade citocromo c de la mitocondria al citosol noestáclaro, pero parece establecerse una interacción entre $\mathrm{Bcl}-2$, Bcl-x y Bax, y los canales iónicos dependientes de voltaje que controlan la salida de citocromoc. Estudios de fraccionamientocelularhan mostradoausenciade traslocación de Bax del citosol a las membranas mitocondriales, así como ausencia de expresión de citocromo c en la fracción citosólica en ratas tratadas con ácido kaínico [Puig B, Ferrer I, datos no publicados]. Tomados en su conjunto, estos datos apoyan la respuesta de los miembros de la familia Bcl-2 frente a la administración de ácido kaínico, particularmente manifestada por un incre- mentode Bax, juntoauna ausencia de activación de la vía mitocondrial en este modelo de excitotoxicidad.

Estudios recientes han mostrado activación de las vías de proteincinasas activadas por mitógenos (MAPK), del tipo cinasas reguladas por señales extracelulares (ERK1 y ERK2) en el modelo de convulsiones y excitotoxicidad por administración sistémicade ácido kaínico. La activación de estacascada se produce vía Ras, comose demuestramedianteel análisis de activación de Ras p21, y comporta la fosforilación de ERK1 y ERK2 (MAPKP) [74]. Estudios bioquímicos preliminares señalaron la activación de $J N K-1$, la fosforilación de c-Jun y la reducción de ERK y de cinasa de $38 \mathrm{kDa}$ (p-38) después de la inyección de ácido kaínico [75]. Sin embargo, estudios inmunohistoquímicos más detallados han puesto de manifiesto diferencias regionales sustanciales y aumento de las formas fosforiladas. Así, se ha demostrado un aumento de la expresión de las formas fosforiladas (activas) de cinasas activadas por estrés (SAPK/JNK: JNKP) y de la forma fosforilada de $\mathrm{p} 38$ (p38P). MAPKP y JNKP se expresan a las tres y a las seis horas en el hipocampo propio y en la circunvolución dentada. Estos cambios se siguen de la fosforilación de sustratos específicos de estas cinasas. c-MycP y c-JunP se expresan del mismo modo en el hipocampo propio y en la circunvolución dentada, mientras que la expresión de CREBP se reduce en las poblaciones vulnerables del hipocampo propio, pero se conserva en la circunvolución dentada. ATF-2Py Elk-1P se sobreexpresan únicamente en la circunvolución dentada. Estos datos indican que el mantenimiento de CREBPy la sobreexpresión de ATF-2P y Elk-1P se asocian con supervivencia celular, mientras que el destino delas neuronas que tienen expresión aumentada dec-MycP y c-JunP depende de otros factores que condicionan su progresión hacia la muerte celular o hacia la supervivencia [74].

Estos resultadosnocontradicen otras observacionesqueindican aumento de expresión de $J N K$ y de formas fosforiladas de c-Jun en poblaciones destinadas a morir. Tampococontradicenel posible papel de c-Jun en la supervivencia de ciertas poblaciones neuronales. Sin embargo, sí matizan que la sobreexpresión de estos factores no lleva inexorablemente a la muerte neuronal en el modelo de excitotoxicidad por ácido kaínico. Finalmente, es preciso señalar que ratones nulos para el gen $J N K-1$ son vulnerables al daño excitotóxico, mientras que los ratones nulos para $J N K-3$ son resistentes, lo que indica que $J N K-1$ media en el daño excitotóxicoporácidokaínico[76].

Estudios con microscopía electrónica en las fases tempranas deconvulsionesinducidas experimentalmentemuestran dilatación del citoplasma de los astrocitos perivasculares y perineuronales. Ello se relaciona estrechamente con la entrada y la acumulación decalcio en las mitocondrias de las dendritas basales y en el soma de las neuronas de CA 1 y CA3 pocas horas después de la administracióndeácidokaínico,con recuperaciónalos 40minutos del término de las convulsiones tras la administración de diacepam. También existe entrada de calcio en astrocitos y la recuperación es más lenta. Estas modificaciones son semejantes en distintos modelos de convulsiones en la rata $[77,78]$.

El modo como mueren las neuronas después del daño excitotóxico ha constituido motivo de controversia. Clásicamente se ha consideradolamuerte excitotóxica comonecrosis secundariaala entrada masiva de calcio al interior de las células [79-81]. Sin embargo, estudios posteriores han mostrado la presencia de un componente apoptótico. Estaúltimainterpretación se basa en tres aspectos. Por una parte, en la demostración bioquímica de fragmentos múltiples de 180-200 pares de bases, los cuales sugieren 
activación temprana de endonucleasas y rotura del ADN en los espacios internucleosomales, más vulnerables al no estar cubiertos de histonas. Por otra parte, las células destinadas a morir se tiñen con el método de marcaje in situ del ADN fragmentado, originariamente descrito como específico para la detección de apoptosis in situ [82,83]. El tercer aspecto a favor de apoptosis se basa en la acumulación nuclear de p53 en neuronas vulnerables al ácido kaínico [84].

Sin embargo, los estudios con microscopio electrónico muestran un patrón que difiere de la apoptosis clásica. Las neuronas lesionadas presentan cambios en la membrana nuclear y en las membranas del citoplasma, incluyendolas del retículo endoplásmico rugoso y las del aparato de Golgi; asimismo, muestran alteraciones mitocondriales y disolución de los ribosomas. Finalmente, las células dañadas sólo presentan modificaciones en el núcleo, consistentes en la agregación granular de la cromatina en fases avanzadas. Las imágenes de apoptosis, caracterizadas por condensación extrema de la cromatina y formación de cuerpos apoptóticos con relativa preservación del citoplasma, se observan excepcionalmente en el modelo de excitotoxicidad por administración sistémica de ácido kaínico [85]. Además, diferentes estudios han mostrado que el método de marcaje in situ del ADN fragmentado no es específico de apoptosis, ya que la incorporación de nucleótidos marcados a los terminales 3'-OH del ADN fragmentado mediante el enzima deoxi-nucleotidil-transferasa terminal puede sucederen otras formas de muerteneuronal [86-88]. La expresión aumentada de p53 en el núcleo no necesariamente indica apoptosis.

Recientemente se ha conocido que las caspasas, cisteína proteasas que rompen uniones aspártico, desempeñan un importante papel en la muerte por apoptosis. Las caspasas comprenden miembros que tienen funciones inductoras, como la caspasa- 8 y la caspasa-9, y miembros con funciones ejecutoras, comola caspasa-3 y lacaspasa-7.Lacaspasa-3 puede activarse indistintamente a través de la vía Fas/Fas-L y caspasa-8, o a través de la vía mitocondrial por liberación de citocromo c y unión a Apaf-1, complejo que activa caspasa-9. Las caspasas se expresan de formainactiva como procaspasas en el citoplasma de las células. La activación de caspasa-3-y también la de caspasa-7-comporta la actuación sobre distintos sustratos vitales. La destrucción de estos sustratos por múltiples cortes deriva en la muerte por apoptosis. Unodelos sustratos es poli(ADP-ribosa) polimerasa(PARP), enzima de la membrana nuclear que se rompe específicamente por acción de la caspasa-3 y de la caspasa-7 en dos fragmentos de 89 y $27 \mathrm{kDa}$. La presencia de fragmentos de PARP de $89 \mathrm{kDa}$ es, por tanto, un marcador de la función de las caspasas efectoras o ejecutoras.

Estudios en el modelo de excitotoxicidad por ácido kaínico intraperitoneal han mostradoinducción de ARNm de caspasa-3y aumento de la expresión de procaspasa-3 en algunas neuronas de las regiones vulnerables del hipocampo [89-91]. Más aún, unas pocasneuronas expresan caspasa-3 activa(caspasa-3 hendidade $17 \mathrm{kDa}$ ) y activación de caspasa-3 [92]. Esto indica la participación de la vía de caspasas en ciertas neuronas del hipocampo propio y apuntan hacia la muerte celular con componente apoptótico en subpoblaciones del hipocampo. Sin embargo, los estudios con Western blot señalan la presencia de banda sdePARPde $89 \mathrm{kDa}$ y también de bandas de menor tamaño $(67 \mathrm{kDa})$, lo que indica que la fragmentación dePARP no se produce exclusivamente por activación de caspasas, sino también por actuación de otras proteasas, comopredicelamuerteindiscriminadapornecrosis [91].
Debe tenerse en cuenta que las neuronas son células sociales que precisan de componentes suministrados por otras células para su mantenimiento y desarrollo. Se ha observado una inducción anormal de ARNm para miembros de la familia del factor de crecimiento fibroblástico $-\alpha \mathrm{FGF}$ y $\beta \mathrm{FGF}-\mathrm{y}$ de sus receptores-Flgdespués de lainducción de convulsiones porácidokaínicoen larata [93], así como para los ARNm del factor trófico derivado de glia (GDNF)y de su receptor [94]. Otros factores son neurotrofinas que actúan sobre receptores específicos tirosincinasas.Elfactorderivado del cerebro(BDNF) se encuentra ampliamente distribuido por el sistemanerviosoy, además de suimportancia durante eldesarrollo, mantienela actividad sináptica y el trofismo de distintas poblacionesneuronales enlavidaadulta.ElBDNFactúaespecíficamente sobrelos receptores TrkB, consistentes enformas enterasconel dominio intracelular-que contiene el componente catalítico-y formastruncadas, quecarecendeldominiointracelulary, portanto, defunción de cinasa. Elpapel de estos últimos receptores parece ser eldeneutralizarobloquear laacción deBDNFenlugares dondeno se precisa o es poco apropiada su función.

Estudios de ARNm han mostrado unainducción de BDNFen el hipocampoy de TrkB en la circunvolución dentadaentrelos 30 minutos y las cuatro horas después de la administración de ácido kaínico [95]. Sin embargo, en animales sometidos a inyección intraperitoneal de ácido kaínico en dosis convulsionantes, la expresión de BDNF se pierde en las células que van a morir en el hipocampo propio, pero permanece en poblaciones resistentes. El BDNF sólo se sobreexpresa transitoriamente en las células granulares de la circunvolución dentada seis horas más tarde de la administración de ácido kaínico [96]. La expresión del ARNm para TrkB y de proteína TrkB entera sigue un patrón semejante con reducción en poblaciones que van a perecer y aumento en poblaciones resistentes, particularmente en las prolongaciones de las células granulares de la circunvolución dentada [96,97]. Finalmente, TrkB truncado se expresa en astrocitos reactivos del stratum oriens y del stratum radiatumdel hipocampo, territorios en dondelapérdidadeneuronas obstaculizacualquierintentode establecer posibles conexiones mediante fibras distantes convergentes en un foco sin dianas funcionales [96].

Este escenario apunta a un papel protector del BDNF, que es compatible con el ya conocido para el BDNFen otros modelos, y sugiere la posible utilización del BDNF como agente terapéuticoeneldañoexcitotóxicoporácidokaínico. Seconoceelefecto protector delBDNFen modelos murinos de isquemia global y de isquemia focal. Asimismo, el BDNF protege a las neuronas cerebelosas, hipocampales, cerebro-corticales y septales del daño excitotóxico $[98,99]$. Sin embargo, el resultado dela administración del BDNF es drásticamente opuestoin vivo. El BDNF no tiene un efecto protector frente al ácido kaínicoy, por el contrario, agrava el daño excitotóxico [100].

Como resultado del daño excitotóxico, las fibras musgosas procedentes de las células granulares de la circunvolución dentada se encuentran desconectadas de sus dianas. Esta diferenciación da lugar a la producción de ramitas axonales musgosas que progresan en la región supragranular y en toda la capa molecular de lacircunvolución dentada [101,102]. La formación de ramitas o sprouting se asocia con un incremento de la expresión de la proteína GAP-43 en la capa supragranular durante la primera semana, y en toda la capa molecular a partir de un mes [103]. Asimismo, se ha detectadoincremento de la expresión de la proteína asociada al sinaptosoma de 25 kDa(SNAP-25) en las neuronas y en la capa molecular de la circunvolución dentaday en las 
fibras musgosas del hipocampo en los días siguientes a la lesión excitotóxica porácidokaínico [104-106].Parece probablelaintervención de señales tróficas específicas en el desarrollo de estas conexiones aberrantes, aunque todavíaresulta discutibleelpapel desempeñado por distintos factores tróficos. La presencia del
BDNF y de TrkB en las neuronas de la circunvolución dentada apoya una posible influencia sobre el trofismo de estas células en la construcción de ramitas plásticas dirigidas a reinervar zonas destruidas por el ácido kaínico [96]. Sin embargo, el rebrote de fibras musgosas no se altera en ratones nulos para el BDNF [107].

\section{BIBLIOGRAFÍA}

1. Honavar M, Meldrum BS. Epilepsy. In Graham I, Lantos PL. Greenfield's neuropathology. Vol. 1. London: Arnold; 1997. p. 931-71.

2. De Lanerolle NC, Kim JH, Robbins SJ, Spencer DD. Hippocampal interneuron loss and plasticity in human temporal lobe epilepsy. Brain Res 1989; 495: 387-95.

3. Robbins RJ, Brines ML, Kim JH, Adrian T, De Lanerolle N, Welsh S, et al. A selective loss of somatostatin in the hippocampus of patients with temporal lobe epilepsy. Ann Neurol 1991; 29: 325-32.

4. Sloviter RS, Sollas AL, Barbaro NM, Laxer KD. Calcium-binding protein (calbindin-D28k) and parvalbumin immunocytochemistry in the normal and epileptic hippocampus. J Comp Neurol 1991; 308: 381-96.

5. Babb TL, Pretorius JK, Kupfer WR, Crandall PH. Glutamate decarboxylase immunoreactive neurons are preserved in human epileptic hippocampus. J Neurosci 1989; 9: 2562-74.

6. Green RC, Blume HW, Kupferschmid SB, Mesulam MM. Alterations of hippocampal acetylcholinesterase in human temporal lobe epilepsy. Ann Neurol 1989; 26: 347-51.

7. Johnson EW, De Lanerolle NC, Kim JH, Sanderasan S, Spencer DD, Mattson RH, et al. Central and peripheral benzodiazepine receptors: opposite changes in human epileptogenic tissue. Neurology 1992; 42: 811-5.

8. Wolf HK, Spänle M, Müller MB. Hippocampal loss of GABA $A_{A}$ receptor (1 subunit in patients with chronic pharmacoresistant epilepsies. Acta Neuropathol 1994; 88: 313-9.

9. De Lanerolle NC, Sundaresan S, Brines ML. Distribution of NMDA, quisqualic acid and kainic acid receptors in the hippocampus in human temporal lobe epilepsy. Epilepsia 1990; 31: 625

10. De Lanerolle NC, Brines M, Williamson A, Kim JH, Spencer DD. Neurotransmitters and their receptors in human temporal lobe epilepsy. Epilepsy Res 1992; 7 (Suppl): 235-50.

11. De Lanerolle NC, Brines ML, Kim JH, Williamson A, Philips MF, Spencer DD. Neurochemical remodeling of the hippocampus in human temporal lobe epilepsy. Epilepsy 1992; 9: 205-19.

12. De Lanerolle NC, Eid T, Von Campe G, Kovacs I, Spencer DD, Brines M. Glutamate receptor subunits GluR1 and GluR2/3 distribution shows reorganization of the epileptogenic hippocamopus. Eur J Neurosci 1998; 10: 1687-703.

13. Geddes JW, Cahan LD, Cooper SM, Kim RC, Choi BH, Cotman CW. Altered distribution of excitatory amino acid receptors in temporal lobe epilepsy. Exp Neurol 1990; 108: 214-20.

14. McDonald JW, Garofalo EA, Hood T. Altered excitatory and inhibitory amino acid receptor binding in hippocampus in patients with temporal lobe epilepsy. Ann Neurol 1991; 29: 529-41.

15. Hosford DA, Crain BJ, Cao Z, Bonhaus DW, Friedman AH, Okazaki MM, et al Increased AMPA-sensitive quisqualic recepetor binding in epileptic human hippocampus. J Neurosci 1991; 11: 428-34.

16. Babb TL, Kupfer WR, Pretorius JK. Recurrent excitatory circuits by 'sprouted' mossy fibers into the fascia dentata of human hippocampal epilepsy. Epilepsia 1988; 29: 674.

17. Cascino G, Sutula T, Cavazos J, Parada I, Ramírez L. Hippocampal mossy fiber synaptic reorganization in intractable partial epilepsy: a clinicopathological study. Epilepsia 1988; 29: 684.

18. Sutula T, Cascino G, Cavazos J, Parada I, Ramírez L. Mossy fiber synaptic reorganization in the epileptic human temporal lobe. Ann Neurol 1989; 26: 321-30.

19. Babb TL, Kupfer WR, Pretorius JK, Crandall PH, Levesque MF. Synaptic reorganization by mossy fibers in human epileptic fascia dentata. Neuroscience 1991; 42: 351-63.

20. Mikkonen M, Soininen H, Kälviäinen R, Tapiola T, Ylinen A, Vapalahti M, et al. Remodeling of neuronal circuitries in human temporal lobe epilepsy: increased expression of highly polysialylated neural cell adhesion molecule in the hippocampus and the entorhinal cortex. Ann Neurol 1998; 44: 923-34.

21. Mattern GW, Babb TL, Micevych PE, Blanco CE, Pretorius JK. Granule cell mRNA levels for BDNF, NGF, and NT-3 correlate with neuronal losses and supragranular mossy fiber sprouting in the chronically damaged and epileptic human hippocampus. Mol Chem Neuropathol 1997; 30: 53-76.
22. Hashimoto K, Watanabe K, Nishimura T, Iyo M, Shirayama Y, Minabe Y. Behavioral changes and expression of heat shock protein hsp-70 mRNA, neurotrophic factor mRNA, and cyclooxygenase- 2 mRNA in rat brain following seizures induced by systemic administration of kainic acid. Brain Res 1998; 804: 212-23.

23. Takahashi M, Hayashi S, Kakita A, Wakabayashi K, Fukuda M, Kameyama S, et al. Patients with temporal lobe epilepsy show an increase in brain-derived neurotrophic factor protein and its correlation with neuropeptide Y. Brain Res 1999; 818: 579-82.

24. Murray KD, Isackson PJ, Eskin TA, King MA, Montesinos SP, Abraham LA, et al. Altered mRNA expression for brain-derived neurotrophic factor and type II calcium/calmodulin dependent protein kinase in the hippocampus of patients with intractable temporal lobe epilepsy. J Comp Neurol 2000; 418: 411-22.

25. Jankowski JL, Patterson PH. The role of cytokines and growth factors in seizure and their sequelae. Progr Neurobiol 2001; 63: 125-49.

26. Binder DK, Croll SD, Gall CM, Scharfman HE. BDNF and epilepsy: too much of a good thing? Trends Neurosci 2001; 24: 47-53.

27. Zhu WJ, Roper SN. Brain-derived neurotrophic factor enhances fast excitatory synaptic transmission in human epileptic dentate gyrus. Ann Neurol 2001; 50: 188-94.

28. Schwob JE, Fuller T, Price JL, Olney JW. Widespread patterns of neuronal damage following systemic or intracerebral injections of kainic acid: a histological study. Neuroscience 1980; 5: 991-1014.

29. Lothman EW, Collins RC. Kainic acid-induced limbic seizures: metabolic, behavioral, electroencephalographic and neuropathological correlates. Brain Res 1981; 218: 299-318.

30. Ben-Ari Y, Tremblay E, Riche D, Ghilini G, Naquet R. Electrographic, clinical and pathological alterations following systemic administration of kainic acid, bicuculline and penetrazole: metabolic mapping using the deoxyglucose method with special reference to the pathology of epilepsy. Neuroscience 1981; 6: 1361-91.

31. Sperk G, Lassmann H, Baran H, Kish SJ, Seitelberger F, Honykiewicz O. Kainic acid-induced seizures: neurochemical and histopathological changes. Neuroscience 1983; 10: 1301-15.

32. Sperk G, Lassmann H, Baran H, Seitelberger F, Honykiewicz O. Kainic acid-induced seizures: dose-relationship of behavioural, neurochemical and histopathological changes. Brain Res 1985; 338: 289-95.

33. Ben-Ari Y. Limbic seizure and brain damage produced by kainic acid: mechanisms and relevance to human temporal lobe epilepsy. Neuroscience 1985; 14: 375-403.

34. Lothman EW, Bertram E, Stringer JL. Functional anatomy of hippocampal seizures. Progr Neurobiol 1991; 37: 1-82.

35. Clifford DB, Olnaey JW, Benz AM, Fuller TA, Zorumski CF. Ketamine, phencycline, and MK-801 protect against kainic acid-induced seizurerelated brain damage. Epilepsia. 1990; 31: 382-90.

36. Gass P, Herdegen T, Bravo R, Kiessling M. Spatiotemporal induction of immediate early genes in the rat brain after limbic seizures: effects of NMDA receptor antagonist MK-801. Eur J Neurosci 1993; 5: 933-43.

37. Guarnieri T, Virgili M, Villani L, Facchinetti F, Contestabile A, Migani P. Pharmacological manipulation of the NMDA receptor differentially protects from systemic kainic acid neuropathology: evaluation through ornithine decarboxylase induction, morphology and GFAP immunohistochemistry. Rest Neurol Neurosci 1993; 5: 327-35.

38. Moncada C, Arvin B, Le Peillet E, Meldrum S. Non-NMDA antagonists protect against kainate more than AMPA toxicity in the rat hippocampus. Neurosci Lett 1991; 133: 287-90.

39. Berg M, Bruhn T, Johansen FF, Diemer NH. Kainic acid-induced seizures and brain damaage in the rat: different effects of NMDA and AMPA receptor antagonists. Pharmacol Toxicol 1993; 73: 262-53.

40. Loscher W, Lehmann H, Behl B, Seemann D, Teschendorf HJ, Hofmann HP, et al. A new pyrroyl-quinoxalinedione series of non-NMDA glutamate receptor antagonists: pharmacological characterization and comparison with NBQX and valproate in the kindling model of epilepsy. Eur J Neurosci 1999; 11: 250-62.

41. Patel S, Meldrum BS, Collins JF. Distribution of (3H) kainic acid binding sites in the rat brain: in vivo and in vitro receptor autoradiography. Neurosci Lett 1986; 70: 301-7. 
42. Tremblay E, Represa A, Ben-Ari Y. Autoradiographic localization of kainic acid binding sites in the human hippocampus. Brain Res 1985; 343: 378-82.

43. Castillo PE, Malenka RC, Nicoll RA. Kainate receptors mediate a slow postsynaptic current in hippocampal CA3 neurons. Nature 1997; 388: 182-6.

44. Frerking M, Malenka RC, Nicoll RA. Synaptic activation of kainate receptors on hippocampal interneurons. Nature Neurosci 1998; 1: 479-86.

45. Mulle C, Sailer A, Perg-Otano I, Dickinson-Anson H, Castillo PE, Bureau I, et al. Altered synaptic physiology and reduced susceptibility to kainate-induced seizures in GluR6-deficient mice. Nature 1998; 392: 601-5.

46. Cossart E, Esclapez M, Hirsch JC, Bernard C, Ben-Ari Y. GluR5 kainate receptor activation in interneurons increase increases tonic inhibition of pyramidal cells. Nature Neurosci 1998; 1: 470-8.

47. Frerking M, Nicoll RA. Synaptic kainate receptors. Curr Opin Neurobiol 2000; 10: 342-51.

48. Ben-Ari Y, Cossart R. Kainate, a double agent that generates seizures: two decades of progress. Trends Neurosci 2000; 23: 580-7.

49. Choi DW. Excitotoxic cell death. J Neurobiol 1992; 23: 1261-76

50. Bading H, Segal M, Sucher NJ, Dudek H, Lipton SA, Greenberg ME. $\mathrm{N}$-methyl-D-aspartate receptors are critical for mediating the effects of glutamate on intracellular calcium concentration and immediate early gene expression in cultured hippocampal neurons. Neuroscience 1995; 64: 653-64.

51. Leist M, Nicotera P. Calcium and neuronal death. Rev Physiol Biochem Pharmacol 1998; 132: 79-125.

52. Sloviter RS. Calcium-binding protein (calbindin-D28k) and parvalbumin immunocytochemistry. Localization in the rat hippocampus with specific reference to the selective vulnerability of hippocampal neurons to seizure activity. J Comp Neurol 1989; 280: 183-96.

53. Sloviter RS. Permanently altered hippocampal structure, excitability and inhibition after experimental status epilepticus in the rat: the 'dormant basket cell' hypothesis and its possible relevance to temporal lobe epilepsy. Hippocampus 1991; 1:41-66.

54. Ferrer I, Planas AM. Induction and distribution of heat shock protein-70 messenger and protein following systemic KA injection in the rat: evidence of protein axonal transport. Neuroscience 1995; 69: 1111-8.

55. Planas AM, Soriano MA, Ferrer I, Rodríguez-Farré ER. KA-induced heat shock protein-70, mRNA and protein expression is inhibited by MK-801 in certain rat brain regions. Eur J Neurosci 1995; 7: 293-304

56. Planas AM, Soriano MA, Estrada A, Sanz O, Martín F, Ferrer I. The heat shock protein response after brain lesions: induction of $72 \mathrm{kDa}$ heat shock protein (cell types involved, axonal transpoprt, transcriptional regulation) and protein synthesis inhibition. Progr Neurobiol 1997; 51: 607-36.

57. Yenari MA, Fink SL, Sun GH, Chang LK, Patel MK, Kunis DM, et al. Gene therapy with HSP72 is neuroprotective in rat models of stroke and epilepsy. Ann Neurol 1998; 444: 584-91.

58. Krebs RA, Feder ME. Deleterious consequences of Hsp70 overexpresion in Drosophila melanogaster larvae. Cell Stress Chaperones 1997; 2: 60-71.

59. Schreiber SS, Tocco G, Najm I, Thompson RF, Baudry M. Cycloheximide prevents kainate-induced neuronal death and c-Fos expression in adult rat brain. J Mol Neurosci 1993; 4: 149-59.

60. Pozas E, Ballabriga J, Planas AM, Ferrer I. Kainic acid-induced excitotoxicity is associated with a complex c-Fos and c-Jun response which does not preclude either cell death or survival. J Neurobiol 1997; 33 : 232-46.

61. Le Gal La Salle G. Long-lasting and sequential increase of c-Fos oncoprotein expression in kainic acid-induced status epilepticus. Neurosci Lett 1988; 88: 127-30.

62. Popovici T, Represa A, Crépel A, Barbin G, Beaudoin M, Ben-Ari Y. Effects of kainic acid-induced seizures and ischemia on c-Fos-like proteins in rat brain. Brain Res 1990; 536: 183-94.

63. Gass P, Herdegen T. Neuronal expression of AP-1 proteins in excitotoxic-neurodegenerative disorders and following nerve fiber lesions. Progr Neurobiol 1995; 47: 257-90.

64. Kasof GM, Mandelzys A, Maika SD, Hamer RE, Curran T, Morgan JI. Kainic acid-induced neuronal death is associated with DNA damage and a unique immediate-early gene response in c-Fos-lacZ transgenic rats. J Neurosci 1995; 15: 4238-49.

65. Dragunow M, Preston K. The role of inducible transcription factors in apoptotic cell death. Brain Res Rev 1995; 21: 1-28.

66. Kiessling M, Gass P. Immediate early gene expression in experimental epilepsy. Brain Pathol 1993; 3: 381-93.

67. Tsirka SE, Gualandris A, Amaral DG, Strickland S. Excitotoxin-induced neuronal degeneration and seizure are mediated by tissue plasminogen activator. Nature 1995; 377: 340-4.

68. Tsirka S, Rogove AD, Strickland S. Tissue plasminogen activator and neuronal death. Nature 1996; 384: 123-4.

69. Tsirka S, Rogove AD, Bugge TH, Degon JL, Strickland S. An extracellular proteolytic cascade promotes neuronal degeneration in the mouse hippocampus. J Neurosci 1997; 17: 543-52.

70. Chen ZL, Strickland S. Neuronal death in the hippocampus is promoted by plasmin-catalyzed degradation of laminin. Cell 1997; 91 : 917-25.

71. Tan Z, Levid J, Schreiber SS. Increased expression of Fas (CD95/ APO-1) in adult rat brain after kainate-induced seizures. NeuroReport 2001; 12: 1979-82.

72. Guillardon F, Wichert H, Zimmermann M. Up-regulation of Bax and down-regulation of $\mathrm{Bcl}-2$ is associated with kainate-induced apoptosis in mouse brain. Neurosci Lett 1995; 192: 85-8.

73. López E, Pozas E, Rivera R, Ferrer I. Bcl-2, Bax and Bcl-x expression following kainic acid administration at convulsant doses in the rat. Neuroscience 1999; 91: 1461-70.

74. Ferrer I, Blanco R, Carmona M, Puig B, Domínguez I, Viñals F. Active, phosphorylation-dependent MAP kinases, MAP/ERK, SAPK/JNK and $\mathrm{p} 38$, and specific transcription factor substrates are differentially expressed following systemic administration of kainic acid to the adult rat. Acta Neuropathol 2001 (submitted).

75. Mielke K, Brecht S, Dorst A, Herdegen T. Activity and expression of $J N K-1, \mathrm{p} 38$ and ERK kinases, c-Jun N-terminal phosphorylation, and c-Jun promoter binding in the adult rat brain following kainate-induced seizures. Neuroscience 1999; 91: 471-83.

76. Yang DD, Kuan CY, Whitmarsh AJ, Rincón M, Zheng TS, Davis RJ, et al. Absence of excitotoxicity-induced apoptosis in the hippocampus of mice lacking the $J N K-3$ gene. Nature 1997; 289: 865-70.

77. Evans MC, Griffiths T, Meldrum BS. Kainic acid seizures and the reversibility of calcium loading in vulnerable neurons in the hippocampus. Neuropathol Appl Neurobiol 1984; 10: 285-302.

78. Griffiths T, Evans MC, Meldrum BS. Status epilepticus: the reversibility of calcium loading and the acute neuronal pathological changes in the rat hippocampus. Neuroscience 1984; 12: 557-67.

79. Ignatowicz E, Vezzani AM, Rizzi M, D'Incalci M. Nerve cell death induced in vivo by kainic acid and quinolinic acid does not involve apoptosis. Neuroreport 1991; 2: 651-4.

80. Fujikawa DG, Shinmei SS, Cai B. Kainic acid-induced seizures produce necrotic, not apoptotic, neurons with internucleosomal DNA cleavage: implications for programmed cell death mechanisms. Neuroscience 2000; 98: 41-53.

81. Fujikawa DG, Shinmei SS, Cai B. Seizure-induced neuronal necrosis: implications for programmed cell death mechanisms. Epilepsia 2000; 41: 9-13.

82. Filipkowski R, Hetman M, Kaminska B, Kaczmare KL. DNA fragmentation in rat brain after intraperitoneal administration of kainate. Neuroreport 1994; 5 : 1538-40.

83. Pollard H, Charriaut-Marlangue C, Cantagrel S, Represa A, Robain $\mathrm{O}$, Moreau J, et al. Kainate-induced apoptotic cell death in hippocampal neurons. Neuroscience 1994; 6: 7-18.

84. Sakhi S, Sun N, Wing LL, Mehta P, Schreiber SS. Nuclear accumulation of p53 protein following kainic acid-induced seizures. Neuroreport 1996; 7: 493-6.

85. Domínguez I, Blasco-Ibáñez JM, Ferrer I, Martínez-Guijarro FJ. Typology of the neuronal damage in the hippocampus of mice treated with kainic acid and diethylditiocarbamate. Brain Res (submitted).

86. Ferrer I, Tortosa A, Macaya A, Sierra A, Moreno D, Munell F, et al. Evidence of nuclear DNA fragmentation following hypoxia-ischemia in the infant rat brain, and transient forebrain ischemia in the adult gerbil. Brain Pathol 1994; 4: 115-22.

87. Charriaut-Marlangue C, Ben-Ari Y. A cautionary note on the use of the TUNEL stain to determine apoptosis. Neuroreport 1995; 7: 61-4.

88. De Torres C, Munell F, Ferrer I, Reventós J, Macaya A. Identification of necrotic cell death by the TUNEL assay in the hypoxic-ischemic neonatal rat brain. Neurosci Lett 1997; 230: 1-4.

89. Becker AJ, Guillardon F, Blümcke I, Langenndörfer D, Beck H, Wiestler OD. Differential regulation of apoptosis-related genes in resistant and vulnerable subfields of the rat epileptic hippocampus. Mol Brain Res 1999; 67: 172-6.

90. Faherty CJ, Xanthoudakis S, Smeyne RJ. Caspase-3-dependent neuronal death in the hippocampus following kainic acid treatment. Mol Brain Res 1999; 70: 159-63.

91. Ferrer I, López E, Blanco R, Rivera R, Krupinski J, Martí E. Differential c-Fos and caspase expression following kainic acid excitotoxicity. Acta Neuropathol 2000; 99: 245-56.

92. Henshall DC, Chen J, Simon RP. Involvement of caspase-3-like pro- 
tease in the mechanism of cell death following focally evoked limbic seizures. J Neurochem 2000; 74: 1215-23.

93. Bugra K, Pollard H, Charton G, Moreau J, Ben-Ari Y, Khrestchalisky M. aFGF, bFGF and Flg mRNAs show distinct patterns of induction in the hippocampus following kainate-induced seizures. Eur J Neurosci 1994; 6: 58-66.

94. Reeben M, Laurikainen A, Hiltunen JO, Castren E, Saarma M. The messenger RNAs for both glial cell line-derived neurotrophic factor GDNF, c-ret and GDNFR $\alpha$ are induced in the rat brain in response to kainate induced excitation. Neuroscience 1998; 83: 151-9.

95. Dugich-Djordjevic MM, Tocco G, Lapehak PA, Pasinetti GM, Najm I, Baudry M, et al. Regionally specific and rapid increases in brain derived neurotrophic factor messenger mRNA in the adult rat forebrain following seizures induced by systemic administration of kainate. Neuroscience 1992; 47: 303-15.

96. Goutan E, Martí E, Ferrer I. BDNF, and full length and truncated TrkB expression in the hippocampus of the rat following kainic acid excitotoxic damage. Evidence of complex time-dependent and cellspecific responses. Mol Brain Res 1998; 59: 154-64.

97. Dugich-Djordjevic MM, Ohsawa F, Okazaki T, Mori N, Day JR, Beck $\mathrm{KD}$, et al. Differential regulation of catalytic and non-catalytic trkB messenger RNAs in the rat hippocampus following seizures induced by systemic administration of kainic acid. Neuroscience 1995; 66: 861-77.

98. Lindholm D, Dechant G, Heisenberg CP, Thoenen H. Brain-derived neurotrophic factor is a survival factor for cultured rat cerebellar granule neurons and protects them against glutamate-induced neurotoxicity. Eur J Neurosci 1993; 5: 1455-64.

99. Cheng B, Matson MP. NT-3 and BDNF protect CNS neurons against metabolic/excitotoxic insults. Brain Res 1994; 640: 56-7.

\section{SEÑALIZACION CELULAR}

\section{EN EL HIPOCAMPO EPILÉPTICO}

Resumen. La señalización celular que manda sobre la muerte o la supervivencia en el hipocampo epiléptico humano es difícil de identificar a causa del prolongado intervalo entre el comienzo de los síntomas y el muestreo del tejido cerebral dañado para el examen neuropatológico. La inyección intraperitonial del glutamato análogo del ácido kaínico (AK) es una herramienta útil para el análisis de los efectos de convulsiones y el daño excitotóxico en el hipocampo del roedor. El AK actúa sobre los receptores NMDA y AK, mientras que el impacto sobre los receptores AMPA es muy pequeño. Las neuronas del hilio y las neuronas $C A 3$ son dianas primarias del AK, aunque las neuronas gabérgicas que contienen parvoalbúmina son menos vulnerables que las neuronas glutamatérgicas. Las respuestas inmediatas al AK son la inducción de hsp-70, ARNm y la expresión de la proteína HSP-70/72, junto con c-fos y c-jun ARNm, y la expresión de las proteínas $c$-Fos y cJun en el hipocampo, aunque la expresión aumentada de c-Fos y c-Jun no predice la muerte celular o la supervivencia de la misma. Por el contrario, el activador del plasminógeno tisular (tPA) y la vía de señal de la membrana Fas/FasL probablemente tenga un papel facilitador en el seguimiento de la muerte celular por la inyección de AK. La afectación de otras vías permanece controvertida. Un aumento en la expresión de Bas pro-apotótico junto con una reducción en Bcl-2 sugiere apoptosis ayudado por Bax. La activación de la vía mitocondrial incluye el escape del citocromo c al citosol y activación de la cascada caspasa que lleva a la apoptosis. Sin embargo, otros estudios han puesto de relieve la expresión limitada de la caspasa-3, el ejecutor principal de la apoptosis, y la relevancia de necrosis como la forma principal de la muerte celular después de la excitotoxicidad del AK. La activación fosforilación dependiente de varias cinasas, entre ellas MAPK, p38 y JNK/SAPK, y sus sustratos, se ha encontrado en los animales tratados con AK. La expresión disminuida de CREBp se asocia con la muerte celular, mientras que el aumento en ATF-2P y Elk-1P se asocia con la supervivencia celular. Los factores tróficos probablemente no desempeñan un papel significativo en las etapas iniciales del daño hipocampal, pero tienen su importancia en el remodelado de las células granulares y el brote de fibras musgosas a la capa molecular del giro dentado. Esta regeneración anómala , a su vez, facilita el 'reclutamiento' y mantenimiento crónico de las convulsiones. [REV NEUROL 2002; 34: 544-50]

Palabras clave. Ácido kaínico. Apoptosis. Bcl-2. Caspasas. Cinasas. Convulsiones. Fas. HSP-72. Necrosis.
100. Rudge JS, Mather PE, Pasnikowski EM, Cai N, Corcoran T, Acheson A, et al. Endogenous BDNF protein is increased in adult rat hippocampus after kainic-induced excitotoxic insult but exogenous BDNF is not neuroprotective. Exp Neurol 1998; 149: 398-410.

101. Tauck DL, Nadler JV. Evidence of functional mossy fiber sprouting in the hippocampal formation of kainic acid-treated rats. J Neurosci 1985; 5: 1016-22.

102. Cronin J, Dudek FE. Chronic seizures and collateral sprouting of dentate mossy fibers after kainic acid treatment in rats. Brain Res 1988 ; 474: 181-4.

103. Bendotti C, Pende M, Samanin R. Expression of GAP-43 in the granule cells of rat hippocampus after seizure-induced sprouting of mossy fiber: in situ hybridization and immunocytochemical studies. Eur J Neurosci 1994; 6: 509-15.

104. Boschert U, O’Shaughnessy C, Dickinson R, Tessari M, Bendotti C, Catsicas $\mathrm{S}$, et al. Developmental and plasticity-related differential expression of two SNAP-25 isoforms in the rat brain. J Comp Neurol 1996; 367: 177-93.

105. Geddes JW, Hess EJ, Hart RA, Kesslak JP, Cotman CW, Wilson MC. Lesions of hippocampal circuitry define synaptosomal-associated protein-25 (SNAP-25) as a novel presynaptic marker. Neuroscience 1990; 38:515-25.

106. Martí E, Blasi J, Gómez de Aranda I, Ribera R, Blanco R, Ferrer I. Selective early induction of synaptosomal-associated protein (molecular weigth 25,000 ) following systemic administration of kainate at convulsant doses in the rat. Neuroscience 1999; 90: 1421-32.

107. Bender R, Heimrich B, Meyer M, Frotscher M. Hippocampal mossy fiber sprouting is not impaired in brain-derived neurotrophic factor deficient mice. Exp Brain Res 1998; 120: 399-402.

\section{SINALIZAÇÃO CELULAR \\ NO HIPOCAMPO EPILÉPTICO}

Resumo. A sinalização celular que comanda a morte ou a sobrevivência no hipocampo epiléptico humano é difícil de ser traçada devido ao longo intervalo entre o início dos sintomas e a amostragem de tecido cerebral lesionado para exame neuropatológico. A injecção intraperitoneal de ácido caínico (AC), um análogo do glutamato, é uma ferramenta útil para analisar os efeitos das convulsões e da lesão citotóxica no hipocampo de roedores. $O$ AC actua sobre os receptores NMDA e AC, enquanto que tem um impacto reduzido sobre os receptores AMPA. Os neurónios do hilo e os neurónios CA3 são os alvos primários do AC, apesar dos neurónios gabaérgicos, contentores de parvalbumina, serem menos vulneráveis do que os neurónios glutamatérgicos. As respostas imediatas ao AC são a indução $m R N A$ hsp-70 e a expressão da proteína HSP-70/72, bem como mRNA c-fos e c-jun, e a expressão da proteína c-Fos e c-Jun no hipocampo. Logo, o aumento da expressão c-Fos e c-Jun não é um indicador da morte ou da sobrevivência da célula. Por outro lado, o activador do plasminogénio tecidular (tPA) e a via sinalizadora da membrana Fas/ Fas-L têm provavelmente um papel favorecedor na morte da célula, após injecção de AC. O envolvimento de outras vias permanece controverso. A expressão aumentada do Bax pró-apoptótico, juntamente com Bcl-2 reduzido, sugere apoptose mediada pelo Bax. A activação da via mitocondrial inclui saída do citocromo c para o citosol $e$ activação da cascata de caspase que conduz à apoptose. Contudo, outros estudos evidenciaram a expressão limitada da caspase-3, o principal executor da apoptose, e a relevância da necrose como forma principal de morte celular após excito-toxicidade AC. A activação dependente da fosforilação de diversas quinases, incluindo a MAPK, p-38 e JNK/SAPK, e respectivos substratos foi encontrada em animais tratados com AC. A expressão reduzida de CREBp está associada à morte celular, enquanto que o ATF-2P e Elk-1P estão associados à sobrevivência celular. Provavelmente, os factores tróficos não desempenham um papel importante durante as fases iniciais de lesão do hipocampo, mas são importantes na remodelação das células granulares e no rebentar das fibras mossy para a camada molecular do núcleo dentado. Esta regeneração anormal, por seu lado, favorece as convulsões e a manutenção crónica das mesmas. [REV NEUROL 2002; 34: 544-50]

Palavras chave. Ácido caínico. Apoptose. Bcl-2. Caspases. Convulsões. Fas. HSP-72. Necrose. Quinases. 